\title{
VIOLENCIA ESCOLAR Y NIVELES DE LOGRO DE APRENDIZAJE EN UNA INSTITUCIÓN EDUCATIVA PÚBLICA DE PUERTO MALDONADO
}

\author{
SCHOOL VIOLENCE AND LEVELS OF LEARNING ACHIEVEMENT IN \\ A PUBLIC EDUCATIONAL INSTITUT'TION IN PUERTO MALDONADO \\ (D) Edwin Gustavo Estrada Araoz ${ }^{1 *}$, (D) Helen Juddy Mamani Uchasara ${ }^{1}$ \\ edwin5721@outlook.com; reading.k.vision@gmail.com \\ ${ }^{1}$ Universidad Nacional Amazónica de Madre de Dios, Madre de Dios, Perú
}

*Correspondencia: Edwin Gustavo Estrada Araoz. Email: edwin5721@outlook.com

Recibido: 20.04.2020 | Aprobado: 15.05.2020

\section{RESUMEN}

El objetivo fue establecer la relación que existe entre la violencia escolar y los niveles de logro de aprendizaje de los estudiantes del quinto ciclo de una institución educativa pública de Puerto Maldonado. El enfoque de investigación fue cuantitativo, el diseño no experimental y el nivel relacional. Los instrumentos de recolección de datos fueron el Test AVE y el Acta Consolidada de Evaluación. Los resultados indican que existe una relación inversa y significativa entre la violencia escolar y los niveles de logro de aprendizaje ( $\mathrm{rs}=$ $-0,297 ; \mathrm{p}=0,021<0,05)$. Se concluyó que los altos niveles de violencia escolar están relacionados con los bajos niveles de logro de aprendizaje.

Palabras clave: Violencia escolar, niveles de logro de aprendizaje, educación primaria.

\section{ABSTRACT}

The objective was to establish the relationship between school violence and levels of learning achievement of the fifth cycle students of a public educational institution in Puerto Maldonado. The research focus was quantitative, the nonexperimental design and the relational level. The data collection instruments were the AVE Test and the Consolidated Assessment Act. The results indicate that there is an inverse and significant relationship between school violence and levels of learning achievement $(\mathrm{rs}=-0.297 ; \mathrm{p}=$ $0.021<0.05$ ). It was concluded that high levels of school violence are related to low levels of learning achievement.

Keywords: School violence, levels of learning achievement, primary education. 


\section{INTRODUCCIÓN}

La violencia escolar es un tema que, en la actualidad, ha adquirido mucha importancia, debido a la frecuencia con que se presenta, además de las consecuencias personales y sociales en que deriva (Ayala, 2015), muy a pesar de que existen diversas políticas educativas y sociales, dedicadas a reducirlas y prevenirlas, así como a fomentar una convivencia escolar saludable (Varela, Ávila y Martínez, 2013).

De acuerdo a los datos expuestos en el año 2019 por el Sistema Especializado en Reporte de Casos sobre Violencia Escolar - SISEVE, entre los años 2013 y 2018 se han reportado aproximadamente 14215 casos de violencia entre escolares, lo cual indicaría que existen deficiencias para que los estudiantes se relacionen y solucionen sus problemas de la mejor manera. En esa línea, el $57 \%$ de casos de violencia entre escolares tuvo como víctima a un niño o adolescente hombre y el $43 \%$, a una niña o adolescente mujer (Ministerio de Educación, 2019).

La violencia escolar es conceptualizada como "como cualquier acción que ocurra y se geste en la escuela, dificultando su finalidad educativa y lesionando la integridad de algún miembro de la comunidad escolar" (Pacheco, 2018, p.113). En ese sentido, obstruye el proceso de enseñanza - aprendizaje en las aulas, asimismo, deteriora las relaciones sociales que existen en ella, y agrava el desarrollo de importantes problemas psicológicos y de interacción entre los estudiantes que se encuentran implicados en las dinámicas violentas (Jiménez y Lehalle, 2012).

Por su parte, el Ministerio de Educación (2018) sostiene que la violencia escolar hace referencia a la utilización planificada del poder y la fuerza, ya sea como amenaza o efectivo, contra uno mismo, otro estudiante, un grupo o la comunidad educativa, que provoque o pueda generar contusiones, daños psicológicos, perturbaciones del desarrollo o privaciones. Estas situaciones suelen ocurrir dentro de la escuela o en sus inmediaciones, entre la escuela y el hogar, también mediante el Internet y otros medios de comunicación. Entre los tipos de violencia escolar se encuentran la violencia física, verbal, psicológica, sexual, entre otras.

De acuerdo a Eljach (2011) la violencia escolar puede darse de diversas modalidades, tales como bullying, donde el agresor tiene una ventaja sobre la víctima; violencia verbal, donde se emplea un lenguaje ofensivo, tono de voz agresivo, palabras crueles, tajantes y cortantes; violencia física, que puede ir desde un jalón de orejas, o una cachetada, hasta quemaduras, ahogamientos y el empleo de objetos como correas; violencia psicológica, como amenazar, ignorar, discriminar, humillar, ser indiferente, rechazar y realizar otros comportamientos que pueden generar daños en el desarrollo psicológico; y violencia sexual, que implica intentar consumar un acto sexual, los comentarios o insinuaciones sexuales no deseadas, o las acciones para comercializar o utilizar de cualquier otro modo la sexualidad de una persona a la fuerza.

Con relación a los niveles de logro de aprendizaje, son formulaciones que establecen categorías específicas de desempeño y describen qué son capaces de hacer o resolver los estudiantes en cada nivel; determinan el nivel o el grado de los conocimientos de cada estudiante y se detallan a partir de la evaluación que se les realiza (Cerna, 2016). 
En esa línea, el Ministerio de Educación (2009) sostiene que los niveles de logro indican el grado de desarrollo de las capacidades, conocimientos y actitudes. Se representa mediante calificativos literales que dan cuenta, de modo descriptivo, de lo que sabe hacer y evidencia el estudiante (p.309).

Así, pueden definirse como "los indicios, rasgos, señales, datos e informaciones perceptibles que permiten confirmar logros de aprendizaje en relación con las intenciones de enseñanza y que al ser confrontados con lo esperado pueden considerarse como evidencias significativas del aprendizaje” (Mateo, 2017, p.63).

Actualmente, el Ministerio de Educación (2016) precisa que en el nivel de educación primaria existen 4 niveles de logro: en inicio, cuando el estudiante muestra un progreso mínimo en una competencia de acuerdo al nivel esperado, en el cual evidencia con frecuencia dificultades en el desarrollo de las tareas, por lo que necesita mayor tiempo de acompañamiento e intervención del docente; en proceso, cuando el estudiante está próximo o cerca al nivel esperado respecto a la competencia, para lo cual requiere acompañamiento durante un tiempo razonable para lograrlo; logro esperado, cuando el estudiante evidencia el nivel esperado respecto a la competencia, demostrando manejo satisfactorio en todas las tareas propuestas y en el tiempo programado; y logro destacado, donde el estudiante evidencia un nivel superior a lo esperado respecto a la competencia, es decir, demuestra aprendizajes que van más allá del nivel esperado.

En cuanto a los antecedentes, existen investigaciones que han estudiado la relación existente entre la violencia familiar y los niveles de logro de aprendizaje, las cuales concluyeron que ambas variables se relacionan inversamente, es decir, los estudiantes que se desenvuelven en entornos escolares donde prima la violencia, la agresividad y el acoso escolar tienden a presentar niveles de logro por debajo de lo esperado en comparación a los estudiantes que se desenvuelven en contextos donde la convivencia escolar se basa en el respeto (Calle, Matos y Orozco, 2017; Morcillo, 2012; Alayo y Pinedo, 2016; Ahón, 2017; Quispe y Poma, 2015; Estrada y Mamani, 2019; Martínez, 2019; Cid et al., 2008).

Bajo esa premisa, se planteó como problema de investigación: ¿Qué relación existe entre la violencia escolar y los niveles de logro de aprendizaje de los estudiantes del quinto ciclo de una institución educativa pública de Puerto Maldonado?

Finalmente, para responder al problema se formuló el objetivo general, el cual buscó establecer la relación existente entre la violencia escolar y los niveles de logro de aprendizaje de los estudiantes del quinto ciclo de una institución educativa pública de Puerto Maldonado.

\section{MATERIALES Y MÉTODOS}

El enfoque de investigación fue cuantitativo, ya que la recolección y análisis de datos estuvieron enfocados en contrastar la hipótesis mediante el uso de la estadística (Sánchez, Reyes y Mejía, 2018); el diseño fue correlacional, debido a que las variables de estudio no fueron manipuladas, sino observadas y analizadas (Hernández, Fernández y Baptista, 2014) y el nivel de investigación fue relacional porque se buscó conocer las relaciones existentes entre las variables violencia escolar y niveles de logro de aprendizaje (Bisquerra, 2009). 
La población fue conformada por 502 estudiantes que cursaban el quinto ciclo (quinto y sexto grado de educación primaria) en una institución educativa pública de la ciudad de Puerto Maldonado y la muestra estuvo conformada por 218 estudiantes, cantidad que fue determinada a través de un muestreo probabilístico estratificado. En la tabla 1 se detalla la información expuesta.

\section{Tabla 1}

Distribución de la población y la muestra

\begin{tabular}{lllll}
\hline \multirow{2}{*}{ Grado } & \multicolumn{2}{c}{ Población } & \multicolumn{2}{c}{ Muestra } \\
\cline { 2 - 5 } & $\mathbf{n}$ & $\mathbf{0}$ & $\mathbf{n}$ & $\mathbf{\%}$ \\
\hline Quinto grado & 250 & 49,8 & 108 & 49,8 \\
Sexto grado & 252 & 50,2 & 110 & 50,2 \\
Total & 502 & 100,0 & 218 & 100,0 \\
\hline
\end{tabular}

Fuente: Nómina de matrícula.

La técnica de recolección de datos utilizada para medir la variable violencia familiar fue la encuesta y para la variable niveles de logro de aprendizaje se recurrió a la técnica del análisis documental. Por otro lado, los instrumentos utilizados fueron el Test AVE y el Acta Consolidada de Evaluación.

El Test AVE (Acoso y Violencia Escolar) fue elaborado por Piñuel y Oñate (2006), está conformado por 50 ítems de tipo Likert (nunca, pocas veces, muchas veces) y evalúa 4 dimensiones: hostigamiento (ítems del 1 al 12), intimidación (ítems del 13 al 25), exclusión (ítems del 26 al 42) y agresión (ítems del 43 al 50). Sus propiedades psicométricas fueron halladas a través del proceso de validez y confiabilidad. La validez de contenido fue realizada mediante la técnica de juicio de expertos, para ello se recurrió a 3 psicólogos quienes evaluaron la claridad, coherencia y pertinencia. El coeficiente V de Aiken fue de 0,821 lo cual significa que el test tiene buena validez. Con relación a la confiabilidad, fue determinada mediante una prueba piloto, donde se obtuvo un coeficiente Alfa de Cronbach de 0,835 lo que indica que el test es confiable.

Con relación al Acta Consolidada de Evaluación, consolida la evaluación realizada a los estudiantes en todas las áreas curriculares (Comunicación, Matemática, Personal Social, Ciencia y Tecnología, Educación Física, Arte y Cultura y Educación Religiosa) mediante una evaluación formativa.

La recolección de datos se realizó en el mes de diciembre del año 2019 y estuvo enmarcada en dos momentos. En el primer momento, se gestionó la autorización de la dirección de la institución educativa donde se aplicó los instrumentos, así como a los padres de familia, para que den su consentimiento informado. En la segunda etapa, se aplicó el test y al finalizar el año se solicitó el acta de evaluación para obtener información sobre los niveles de logro de aprendizaje de los estudiantes.

Para desarrollar el análisis estadístico se utilizó el Software SPSS versión 22. Los datos fueron organizados y sistematizados mediante tablas de frecuencia y porcentaje. Para el análisis inferencial se utilizó la prueba no paramétrica rho de Spearman, debido a la naturaleza y nivel de medición de las variables, ya que no se ajustan a la distribución 
normal. Finalmente, para la significancia estadística, se consideró un valor menor o igual a 0,05 .

\section{RESULTADOS}

Los resultados que se muestran a continuación proceden de la recolección de datos efectuadas a los estudiantes del quinto ciclo de una institución educativa pública de la ciudad de Puerto Maldonado.

\section{Tabla 2}

Resultados de la variable violencia escolar

\begin{tabular}{lll}
\hline Categorías & Frecuencia & Porcentaje \\
\hline Muy bajo & 13 & 6,0 \\
Bajo & 39 & 17,9 \\
Moderado & 111 & 50,9 \\
Alto & 41 & 18,8 \\
Muy alto & 14 & 6,4 \\
\hline Total & 218 & 100,0 \\
\hline
\end{tabular}

Fuente: Elaboración propia

En la tabla 2, se puede apreciar que el 50,9\% de estudiantes señalan que el nivel de violencia escolar que perciben es moderado; el 18,8\% indican que el nivel es alto; el 17,9\% señalan que es bajo; el 6,4\% menciona que es muy alto y el $6 \%$ precisa que es muy bajo.

\section{Tabla 3}

Resultados de la variable niveles de logro de aprendizaje

\begin{tabular}{lll}
\hline Categorías & Frecuencia & Porcentaje \\
\hline En inicio & 11 & 5,0 \\
En proceso & 28 & 12,9 \\
Logro esperado & 155 & 71,1 \\
Logro destacado & 24 & 11,0 \\
\hline Total & 218 & 100,0 \\
\hline
\end{tabular}

Fuente: Elaboración propia

De acuerdo a la tabla 3, el 71,1\% de los estudiantes ha logrado los aprendizajes esperados; el $12,9 \%$ está en proceso de lograrlo; el 11\% presenta un logro destacado y el $5 \%$ está en inicio. 


\section{Tabla 4}

Correlación entre la violencia escolar y los niveles de logro de aprendizaje

\begin{tabular}{lllll}
\hline & & & $\begin{array}{l}\text { Violencia } \\
\text { escolar }\end{array}$ & $\begin{array}{l}\text { Niveles de } \\
\text { logro de } \\
\text { aprendizaje }\end{array}$ \\
\hline \multirow{4}{*}{$\begin{array}{l}\text { Rho de } \\
\text { Spearman }\end{array}$} & Violencia & Coeficiente de correlación & 1,000 &,$- 297^{*}$ \\
& escolar & Sig. (bilateral) &. &, 021 \\
& & $\mathrm{~N}$ & 218 & 218 \\
\cline { 2 - 5 } & Niveles de & Coeficiente de correlación &,$- 297^{*}$ & 1,000 \\
& logro de & Sig. (bilateral) &, 021 & $\cdot$ \\
& aprendizaje & $\mathrm{N}$ & 218 & 218 \\
\hline
\end{tabular}

*. La correlación es significativa en el nivel 0,05 (2 colas).

Fuente: Elaboración propia

En la tabla 4, se puede observar que existe una relación inversa y significativa entre la violencia escolar y los niveles de logro de aprendizaje ( $r s=-0,297 ; p=0,021<0,05)$. Ello indica que los altos niveles de violencia escolar están relacionados con los bajos niveles de logro de aprendizaje y viceversa.

\section{Tabla 5}

Correlación entre las dimensiones de la violencia escolary los niveles de logro de aprendizaje

\begin{tabular}{llc}
\hline & & Niveles de logro de aprendizaje \\
\hline \multirow{4}{*}{ Hostigamiento } & Coeficiente de correlación &,$- 201^{*}$ \\
& Sig. (bilateral) &, 034 \\
& $\mathrm{~N}$ & 218 \\
\hline \multirow{3}{*}{ Intimidación } & Coeficiente de correlación &,$- 299^{*}$ \\
& Sig. (bilateral) &, 012 \\
& $\mathrm{~N}$ & 218 \\
\hline \multirow{3}{*}{ Exclusión } & Coeficiente de correlación &,$- 312^{*}$ \\
& Sig. (bilateral) &, 011 \\
& $\mathrm{~N}$ & 218 \\
\hline \multirow{3}{*}{ Agresión } & Coeficiente de correlación &,$- 143^{*}$ \\
& Sig. (bilateral) &, 048 \\
& $\mathrm{~N}$ & 218 \\
\hline
\end{tabular}

*. La correlación es significativa en el nivel 0,01 (2 colas).

Fuente: Elaboración propia

En la tabla 5, se visualiza que existe relación inversa y significativa entre los niveles de logro de aprendizaje y el hostigamiento ( $r s=-0,201 ; \mathrm{p}=0,034<0,05)$; la intimidación $(\mathrm{rs}=-0,299 ; \mathrm{p}=0,012<0,05)$; la exclusión $(\mathrm{rs}=-0,312 ; \mathrm{p}=0,011<0,05)$ y la agresión ( $\mathrm{rs}=$ $0,143 ; \mathrm{p}=0,048<0,05)$. 


\section{DISCUSIÓN}

El objetivo de investigación fue establecer la relación que existe entre la violencia escolar y los niveles de logro de aprendizaje de los estudiantes del quinto ciclo de una institución educativa pública de Puerto Maldonado. Para ello aplicamos el Test AVE y accedimos al Acta Consolidada de Evaluación.

Un hallazgo importante señala que la mayoría de estudiantes precisan que el nivel de violencia escolar que perciben es moderado, lo cual se caracteriza porque a veces se presencian actos deliberados de maltrato verbal y físico contra ellos o sus compañeros dentro del entorno escolar, generados por parte de otros estudiantes los cuales se comportan cruelmente con la intención de someterlos, amilanarlos, intimidarlos o amenazarlos para obtener algo a través del chantaje, atentando así contra su dignidad y sus derechos. Aunque estos comportamientos no son frecuentes tienen una tendencia a volverse comunes, por lo que es necesario que los docentes y padres de familia intervengan para corregirlos oportunamente.

Este hallazgo coincide con la investigación de Quispe y Poma (2015) quienes identificaron que el nivel de acoso escolar era medio, lo cual indicaba que percibían en algunas ocasiones formas multifacéticas de violencia intencionada (física, verbal o psicológica) entre compañeros, la cual se iba haciendo cada vez más frecuente y provocaba en las víctimas un bajo nivel de autoestima, cuadros de ansiedad, dificultando su integración al grupo y normal desempeño académico.

$\mathrm{Al}$ respecto, Ballesteros (2007) trata de explicar la presencia de casos de violencia en las escuelas, señalando que generalmente es arraigada del núcleo familiar donde se desarrolla cotidianamente; del contexto social, donde interactúa con sus amigos y, de la cultura institucional donde estudia, pues es ahí donde muchas veces se dejan pasar por alto dichas actitudes, agravándose con el tiempo. Dicha explicación se basa en la teoría del aprendizaje social desarrollada por Bandura (1973) quien sostiene que la interacción social, especialmente a través del modelado, explica conductas agresivas y violentas en los niños y adolescentes.

Se puede afirmar entonces que la violencia escolar tiende a ser una réplica de la violencia que se observa en la sociedad, siendo la institución educativa un espejo donde se perpetúan dichos problemas sociales. Por otro lado, Tizón (2018) señala que la violencia escolar no solo afecta a los agresores y a las personas agredidas, sino a todos los actores educativos, puesto que el clima escolar se impregna de hostilidad.

Otro hallazgo importante indica que la mayoría de estudiantes ha logrado desarrollar los aprendizajes esperados, es decir, los estudiantes evidencian el nivel esperado respecto a la competencia, demostrando manejo satisfactorio en todas las tareas propuestas y en el tiempo programado. No obstante, casi la quinta parte del total de estudiantes se encuentran en inicio o en proceso de lograr los aprendizajes previstos, lo cual es motivo de preocupación, debido a que es una cantidad significativa por lo que sería pertinente adoptar estrategias para realizar un acompañamiento diferenciado, fomentando así el logro esperado de aprendizajes. 
Este resultado corrobora los hallazgos de Estrada y Mamani (2019) quienes determinaron que el 67,3\% de los estudiantes evidencian el logro de los aprendizajes previstos en el tiempo programado, no obstante, es necesario apoyar a los estudiantes que se encuentran en inicio y proceso para elevar los niveles de logro de aprendizaje de todos los estudiantes y lograr su desarrollo integral.

Una de las principales variables intervinientes para que los estudiantes alcancen satisfactorios niveles de logro de aprendizaje es el clima de aula, es decir, es necesario que exista una atmósfera de respeto y tolerancia traducidos en valores como la confianza, la cooperación, la asertividad y la gestión del conocimiento, es decir, existan condiciones, además de estructurales, psicológicas para aprender (Sandoval, 2014). Ello coadyuvará a que tengan un desempeño académico adecuado y mejorará, posteriormente, la calidad educativa de la institución.

Con relación al contraste de hipótesis, se estableció que existe una relación inversa y significativa entre la violencia escolar y los niveles de logro de aprendizaje, lo cual indica que los estudiantes implicados como víctimas o agresores en casos de violencia física o psicológica, tanto dentro como en las inmediaciones de la escuela tienden a presentar niveles de logro que presenten menores en comparación a contextos donde la convivencia escolar es adecuada.

Hallazgos similares fueron obtenidos por Alayo y Pinedo (2016) quienes determinaron que existe asociación entre acoso escolar y violencia y rendimiento académico, lo cual quiere decir que los estudiantes que reciben agresiones tales como burlas, golpes o mofas por su aspecto físico y son amenazados, frecuentemente, por parte de su agresor provoca en las víctimas el desinterés e indiferencia hacia las actividades académicas; en ese sentido, los estudiantes que son víctimas presentan deficiencias en su aprendizaje y como consecuencia presentan bajos niveles de logro de aprendizaje.

Los comportamientos que denotan agresividad y violencia afectan la atmósfera psicológica de la institución educativa y repercute, desfavorablemente, en el aprendizaje de los estudiantes. Por ello, el equipo directivo, docentes y padres de familia deben trabajar conjuntamente para fomentar un buen clima escolar que favorezca el proceso de aprendizaje, basados especialmente en 3 vertientes: el fortalecimiento de los lazos amicales, la no violencia y la búsqueda de perturbaciones para desempeñarse (Ascorra, Arias y Graff, 2003).

En esa misma línea Calle, Matos y Orozco (2017) aseveran que las víctimas de violencia dentro de una institución educativa presentan múltiples consecuencias negativas, tales como presentar bajos niveles de autoestima, incremento de la inseguridad personal, prevalencia de problemas psicológicos, disminución de las oportunidades de aprendizaje, así como bajos niveles de logro de aprendizaje.

Otro hallazgo que llama la atención es que se halló la existencia de una relación inversa y significativa entre los niveles de logro de aprendizaje y el hostigamiento, la intimidación, la exclusión y la agresión. En ese sentido, los factores de la violencia escolar, manifestados física, verbal o psicológicamente están asociados al desempeño académico 
que presenten las víctimas, e incluso, los observadores, ya que generan zozobra, temor y poca predisposición para atender a las clases.

Estos resultados coinciden con la investigación de Cid et al. (2008) quienes señalan que la violencia escolar "es un problema actual y creciente de salud en la comunidad escolar, siendo de tal intensidad que ha provocado incidentes negativos en niños y adolescentes, como dificultad en el aprendizaje y abandono escolar" (p.21).

Los estudiantes, tanto niños como adolescentes, tienen el derecho de educarse para desarrollarse integralmente en ambientes adecuados, seguros y con las condiciones mínimas para lograr aprendizajes. No obstante, en los últimos años los indicadores de violencia en el entorno escolar muestran que la convivencia escolar se ha ido deteriorando, convirtiéndose la escuela en un lugar inseguro donde, frecuentemente, se percibe comportamientos violentos que atentan contra el bienestar psicosocial de los estudiantes que son tanto víctimas como observadores.

En virtud a lo expuesto, es necesario diseñar y rediseñar políticas públicas que fomenten la prevención de toda forma de violencia, partiendo desde el hogar, los espacios públicos y la escuela, pues son lugares donde los estudiantes pasan un tiempo significativo de sus vidas. Asimismo, como medida correctiva se debe implementar tratamiento para los estudiantes que fueron víctimas de violencia escolar, priorizando los casos más graves. Finalmente, sería trascendental que ante la realidad evidenciada se desarrollen programas de prevención e intervención en las instituciones educativas para mejorar la calidad de las relaciones que se establezcan en la escuela y fuera de ella.

\section{CONCLUSIONES}

- La principal conclusión a la que se llegó es que se pudo establecer que existe una relación inversa y significativa entre la violencia escolar y los niveles de logro de aprendizaje de los estudiantes del quinto ciclo de una institución educativa pública de Puerto Maldonado.

- Asimismo, se halló relación inversa y significativa entre los niveles de logro de aprendizaje y las dimensiones hostigamiento, intimidación, exclusión y agresión.

- $\quad$ Por último, se pudo identificar que la mayoría de los estudiantes perciben que el nivel de violencia escolar es medio y han logrado desarrollar los aprendizajes esperados.

\section{REFERENCIAS BIBLIOGRÁFICAS}

Ahón, M. (2017). El acoso escolar y el rendimiento académico en el área de persona, familia y relaciones bumanas en los estudiantes de secundaria de una Institución educativa, Paiján - 2017. (Tesis de posgrado). Universidad César Vallejo, Trujillo, Perú. Recuperado de http:// repositorio.ucv.edu.pe/handle/UCV/18508

Alayo, M. y Pinedo, K. (2016). Bullying y rendimiento académico en el área de comunicación y matemática en los estudiantes de quinto y sexto grado de primaria de una I.E. de Huaycán, 2015. (Tesis de pregrado). Universidad Peruana Unión, Lima, Perú. Recuperado de http://repositorio.upeu.edu.pe/handle/UPEU/179 
Ascorra, P., Arias, H. y Graff, C. (2003). La escuela como contexto de contención social y afectiva. Revista Enfoques Educacionales, 5(1), 117-135.

Ayala, M. (2015). Violencia escolar: un problema complejo. Ra Ximbai, 11(4), 493-509. Recuperado de http://revistas.unam.mx/index.php/rxm/article/view/71358

Ballesteros, M. (2007). Violencia escolar impacto y consecuencia social: propuesta de un programa desde una perspectiva bumanista. (Tesis de posgrado). Universidad Iberoamericana, México D.F., México. Recuperado de http://www.bib.uia.mx/tesis/pdf/014822/ 014822.pdf

Bandura, A. (1973) Aggression: A Social Learning Analysis. NJ: Prentice-Hall.

Bisquerra, R. (2009). Metodologia de la Investigación Educativa. (2a Ed.). Madrid: La Muralla S.A.

Calle, F., Matos, P. y Orozco, R. (2017). El círculo de la violencia escolar en el Perú: hogares, escuela $y$ desempeño educativo. Lima: PUCP. Recuperado de http://repositorio.minedu.gob.pe/handle/MINEDU/5756

Cerna, M. (2016). Nivel de logro de aprendizaje en el área de matemática de los y las estudiantes del IV ciclo de educación básica regular de la Institución Educativa N³2385 "Virgen de Fátima", distrito de Ilata, provincia de Huamalies, región Huánuco, año 2015. (Tesis de pregrado). Universidad Católica los Ángeles de Chimbote, Ancash, Perú. Recuperado de http://repositorio.uladech.edu.pe/handle/123456789/2419

Cid, P., Díaz, A., Pérez, M., Torruella, M. y Valderrama, M. (2008). Agresión y violencia en la escuela como factor de riesgo del aprendizaje escolar. Ciencia y Enfermería, 14(2), 21-30. doi: 10.4067/S0717-95532008000200004

Eljach, S. (2011). Violencia escolar en América Latina y el Caribe: Superficie y fondo. Panamá: UNICEF. Recuperado de http://hdl.handle.net/123456789/1101

Estrada, E. y Mamani, H. (2019). La agresividad y los niveles de logro de los estudiantes del sexto grado de la Institución Educativa "Aplicación Nuestra Señora del Rosario" de Puerto Maldonado. Revista Perspectiva, 20(3), 338-34. doi: 10.33198/rp.v20i3.00047

Hernández, R. Fernández, C. y Baptista, P. (2014) Metodología de la Investigación. (6 ${ }^{\text {ta } e d .) . ~}$ México: Mc Graw-Hill.

Jiménez, T. y Lehalle, H. (2012). La Violencia Escolar entre Iguales en Alumnos Populares y Rechazados. Psychosocial Intervention, 21(1), 77-89. doi: 10.5093/in2012v21n1a5

Martínez, S. (2019). La violencia escolar y su relación con el rendimiento académico en estudiantes de primero de secundaria de las Unidades Educativas Fiscales del Macrodistrito 7 - Centro, de la ciudad de La Paz. (Tesis de grado). Universidad Mayor de San Andrés, La Paz, Bolivia. Recuperado de http://repositorio.umsa.bo/xmlui/handle/123456789/ 22514

Mateo, M. (2017). La Autoestima y el Nivel de Logros de Aprendizaje en Matemática en el Segundo Grado de Secundaria de la Institución Educativa Particular Mentor de San Juan de LuriganchoLima. (Tesis de posgrado). Universidad Nacional de Educación Enrique Guzmán y 
Valle, Lima, Perú. Recuperado de http://repositorio.une.edu.pe/handle/ $\mathrm{UNE} / 1275$

Ministerio de Educación (2009). Diseño curricular nacional de Educación Básica Regular. Lima: MINEDU. Recuperado de http://repositorio.minedu.gob.pe/handle/123456789/ 3738

Ministerio de Educación (2016). Currículo nacional de la educación básica. Lima: MINEDU. Recuperado de http://repositorio.minedu.gob.pe/handle/123456789/4551

Ministerio de Educación (2018). Protocolos para la atención de la violencia escolar. Lima: MINEDU. Recuperado de http://repositorio.minedu.gob.pe/handle/MINEDU/ 5891

Ministerio de Educación (2019). Sistema Especializado en reporte de casos sobre Violencia Escolar - SiseVe. Informe 2013- 2018. Lima: MINEDU. Recuperado de http://repositorio.minedu.gob.pe/handle/MINEDU/6670

Morcillo, B. (2012). La violencia escolar y su influencia sobre el rendimiento académico. (Tesis de posgrado). Universidad Internacional de La Rioja, La Rioja, España. Recuperado de https://reunir.unir.net/handle/123456789/628

Pacheco-Salazar, B. (2018). Violencia escolar: la perspectiva de estudiantes y docentes. Revista Electrónica de Investigación Educativa, 20(1), 112-121. doi: 10.24320/redie.2018.20.1.1523

Piñuel, I. y Oñate, A. (2006). AVE: Acoso y Violencia Escolar. Madrid: Tea Ediciones.

Quispe, J. y Poma, B. (2015). El bullying y el rendimiento académico en los estudiantes de sexto grado de primaria en la institución educativa Francisco Bolognesi No 2016, UGEL 04, Comas, 2014. (Tesis de pregrado). Universidad Nacional de Educación "Enrique Guzmán y Valle”, Lima, Perú. Recuperado de http://repositorio.une.edu.pe/handle/UNE/ 525

Sánchez, H., Reyes, C. y Mejía, K. (2018). Manual de términos en investigación científica, tecnológica y humanistica. Universidad Ricardo Palma: Lima.

Sandoval, M. (2014). Convivencia y clima escolar: claves de la gestión del conocimiento. Última década, 22(41), 153-178. doi: 10.4067/S0718-22362014000200007

Tizón, M. (2018). Perpetuando ciclos de violencia en los colegios: diagnóstico sobre violencia escolar en el Centro Educativo Nacional Max Uble, de Villa el Salvador. Lima. (Tesis de posgrado). Pontificia Universidad Católica del Perú, Lima, Perú. Recuperado de http://hdl.handle.net/20.500.12404/13605

Varela, R., Ávila, M. y Martínez, B. (2013). Violencia escolar: un análisis desde los diferentes contextos de interacción. Psychosocial Intervention, 22(1), 25-32. doi: $10.5093 /$ in $2013 \mathrm{a} 4$

\section{CITAR COMO:}

Estrada Araoz, E. G., \& Mamani Uchasara, H. J. (2020). Violencia escolar y niveles de logro de aprendizaje en una institución educativa pública de Puerto Maldonado. Puriq, 2(3), 165-175. https://doi.org/10.37073/puriq.2.3.86 